\title{
Venomous but not villainous
}

\author{
TAXONOMY \\ PHYLUM: Chordata \\ CLASS: Reptilia \\ ORDER: Squamata \\ SUBORDER: Serpentes
}

\section{Classification}

There are over 3,000 species of snakes found on every continent except Antarctica. Of these, 2,700 comprise the group Caenophidia or 'advanced snakes,' which possess venom-delivery systems or components of such systems. Only about 660 are traditionally considered venomous, including elapids, vipers and asps; these snakes possess tubular front fangs, muscularized venom glands and a bite significantly dangerous to humans. Other caenophidians have venom glands, but their venom does not pose a danger to humans or they lack the fangs necessary for injecting it; these are classified as Colubridae ${ }^{1}$.

\section{Venom}

Snake venoms contain complex mixtures of lethal and pharmacologically active proteins and polypeptides that have evolved to target specific receptors, ion channels or enzymes of prey. These biological molecules effectively perturb vital physiological systems in order to disrupt the movement, circulation and respiration of prey. A single venom can contain more than 100 different proteins, and several hundred proteins have been purified and characterized from snake venoms of various species ${ }^{2}$. In the last several decades, scientists have studied the toxins in snake venoms to determine their mechanisms of action, to find ways to neutralize their toxicity and adverse effects, to develop research tools for the study of normal physiological processes and to develop pharmaceutical agents based on their structures ${ }^{3}$.

\section{Research résumé}

The idea of utilizing purified toxins from snake venoms as a source of therapeutics emerged in the early 20 th century. The first successful drug developed from an isolated toxin was a hypotensive agent called captopril, which was designed in 1975 based on the structure of bradykinin-potentiating peptides (BPPs) isolated from the venom of Bothrops jararaca ${ }^{4}$. Anti-hypertensive agents other than BPPs have been found in the venom of several snake species, including Dendroaspis spp. (such as Dendroaspis angusticeps, also known as the eastern green mamba $)^{4}$. Researchers have also identified antiplatelet agents, pro- and anti-coagulants and fibrinolytic agents (which aid the process of blood clot dissolution). ACE inhibitors, a class of drugs that originated from toxins in snake venom, are currently prescribed to $27 \%$ of patients with hypertension in the US ${ }^{4}$.

At sublethal doses, snake venoms have the potential to reduce the size of solid tumors ${ }^{5}$. Research suggests that cancer cells avoid destruction by the immune system through down-regulation or mutation of death receptors. Finding the agents that increase the expression of death receptors of cancer cells is therefore an important area of research. The snake venom toxin from Vipera lebetina turanica induces the apoptosis of colon cancer cells and also inhibits the growth of human prostate cancer cells and neuroblastoma cells ${ }^{6}$. Scientists recently developed a silica nanoparticlebased snake venom delivery system that targets cancer cells, but not normal cells. When snake venom extracted from Walterinnesia aegyptia was delivered this way, its antitumor activity in two breast carcinoma cell lines and in a multiple myeloma mouse model was strongly enhanced ${ }^{5}$.

Several venom compounds have been used for their antimicrobial effects, including phospholipases A2, metalloproteinases and l-amino acid oxidases ${ }^{7}$. These molecules provide an interesting alternative for controlling microorganisms that are resistant to conventional antibiotics. In another study, proteins isolated from Bothrops leucurus venom were used as antivirals against dengue fever, which does not currently have an approved antiviral therapy available ${ }^{8}$.

1. Vonk, F.J. et al. Snake venom: From fieldwork to the clinic: Recent insights into snake biology, together with new technology allowing high-throughput screening of venom, bring new hope for drug discovery. Bioessays 33, 269-279 (2011).

2. McCleary, R.J. \& Kini, R.M. Non-enzymatic proteins from snake venoms: a gold mine of pharmacological tools and drug leads. Toxicon. 62, 56-74 (2013).

3. Kini, R.M. \& Fox, J.W. Milestones and future prospects in snake venom research. Toxicon. 62, 1-2 (2013).

4. Koh, C.Y. \& Kini, R.M. From snake venom toxins to therapeutics-cardiovascular examples. Toxicon. 59, 497-506 (2012).

5. Al-Sadoon, M.K., Rabah, D.M. \& Badr, G. Enhanced anticancer efficacy of snake venom combined with silica nanoparticles in a murine model of human multiple myeloma: molecular targets for cell cycle arrest and apoptosis induction. Cell Immunol. 284, 129-138 (2013).

6. Park, M.H. et al. Snake venom toxin from Vipera lebetina turanica induces apoptosis of colon cancer cells via upregulation of ROS- and JNK-mediated death receptor expression. BMC Cancer 12, 228 (2012).

7. de Oliveira Junior, N.G., E Silva Cardoso, M.H. \& Franco, O.L. Snake venoms: attractive antimicrobial proteinaceous compounds for therapeutic purposes. Cell Mol. Life Sci. 70, 4645-4658 (2013).

8. Cecilio, A.B. et al. Molecular characterization of Lys49 and Asp49 phospholipases $A_{2}$ from snake venom and their antiviral activities against Dengue virus. Toxins (Basel) 5, 1780-1798 (2013). 